\title{
Late Presentation of Carfilzomib Associated Thrombotic Microangiopathy
}

\author{
Michael Haddadin ${ }^{1}$, Mohammad Al-Sadawi ${ }^{1}$, Sally Madanat ${ }^{2}$, Eric Tam³, \\ Evelyn Taiwo ${ }^{3}$, Carol Luhrs ${ }^{3}$, Samy I Mcfarlane ${ }^{1, *}$ \\ ${ }^{1}$ Department of internal Medicine, State University of New York-Downstate, Brooklyn, New York, USA \\ ${ }^{2}$ Saint James School of Medicine St. Vincent, Cane Hall Road, Arnos Vale, St Vincent \& the Grenadines \\ ${ }^{3}$ Department of Hematology \& Oncology, State University of New York-Downstate, Brooklyn, New York, USA \\ *Corresponding author: Samy.mcfarlane@downstate.edu
}

Received June 17, 2019; Revised July 21, 2019; Accepted July 31, 2019

\begin{abstract}
Multiple Myeloma (MM) is a plasma cell disorder characterized by abnormal proliferation of plasma cells resulting in overproduction of paraprotein. Proteasome inhibitors (PI) have been a corner stone for the treatment of MM. Thrombotic Microangiopathy (TMA) is a recent hematological adverse event that has newly been recognized in multiple PI. TMA leads to end-organ damage and infarction by microthromobi. TMA pathophysiology is not well understood and has multiple etiologies. We present a case of PI-induced TMA, along with literature review of cases diagnosed from 2008-2018. Unique to our case is the onset of presentation, more than 24 months after initiating carfilzomib. Our case highlights the need for vigilant monitoring and the importance of clinical suspicion in patients at risk for TMA.
\end{abstract}

Keywords: carfilzomib, thrombotic microangiopathy, proteosome inhibitos

Cite This Article: Michael Haddadin, Mohammad Al-Sadawi, Sally Madanat, Eric Tam, Evelyn Taiwo, Carol Luhrs, and Samy I Mcfarlane, "Late Presentation of Carfilzomib Associated Thrombotic Microangiopathy." American Journal of Medical Case Reports, vol. 7, no. 10 (2019): 240-243. doi: 10.12691/ajmcr-7-10-5.

\section{Introduction}

Multiple Myeloma (MM) is a plasma cell disorder characterized by abnormal proliferation of plasma cells resulting in overproduction of paraprotein. It represents about $10 \%$ of hematological malignancies with an incidence of 27,000 per year [1]. Treatment often includes chemotherapy and in patients with very good response to chemotherapy, further treatment with autologous hematopoietic stem cell transplant (HSCT) [2].

Proteasome inhibitors (PI) have been a corner stone for the treatment of MM [3,4,5] starting with bortezomib a reversible PI which was approved in 2003, then the introduction of carfilzomib, an irreversible and selective PI, which was approved in 2012. Carfilzomib was initially approved for treatment of refractory MM, but due to higher rates of response and improved survival, it's been increasingly used both as a first-line therapy and at relapse [6].

Well known side effects of PI include peripheral neuropathy, systemic and pulmonary hypertension, heart failure, anemia, fatigue, thrombocytopenia, nausea, pyrexia, dyspnea, diarrhea, headache, and cough [7]. Most grade 3 and 4 adverse effects (medically significant adverse events leading to hospitalization, morbidity or life threatening) are hematological and include thrombocytopenia (23.4\%), anemia (22.4\%), and lymphopenia (18.1\%) [8].
However, a potentially fatal, underreported, and relatively recently discovered adverse effect is thrombotic microangiopathy (TMA) which involves endothelial cell injury and activation of the coagulation cascade, causing thrombocytopenia and microangiopathic hemolytic anemia (MAHA). This cascade of events leads to eventual widespread microvascular thrombosis and end-organ infarction. Interestingly while bortezomib has been present for nearly a decade before carfilzomib, and has broader indications for use, most of the reported bortezomib-induced TMA cases were collected after carfilzomib use, likely due to heightened awareness of this serious complication. Hobeika et al. reported the first case of carfilzomib-induced renal thrombotic microangiopathy on a renal biopsy in 2014 [9].

The reported cases of this phenomenon occurred very soon after initiation of treatment. Here, we report a case of delayed onset carfilzomib-induced TMA occurring after receiving continuous therapy for two years.

\section{Case Presentation}

A 65-year-old-female with IgG-kappa-MM, Revised Multiple Myeloma International Staging System (R-ISS) stage 2, diagnosed 8 years ago. She was initially treated with bortezomib and dexamethasone $(\mathrm{Vd})$ with very good response then an autologous hematopoietic stem cell transplant. Post-transplant, she was treated with lenalidomide maintenance for 13 months but eventually 
had disease progression. Second line treatment with bortezomib, cyclophosphamide and dexamethasone (VCD) was initiated, completed 7 months of treatment. Her disease eventually progressed, and she was switched to carfilzomib, dexamethasone and pomalidomide (KPd). The patient achieved complete remission after 8 cycles of KPd then continued maintenance therapy with carfilzomib monotherapy for one uear until relapse. She was retreated with (KPd), achieving very good partial response after 4 cycles. At patient request, her treatment was de-escalated to carfilzomib and dexamethasone (Kd) only. The MM remained under control, the patient received 2 cycles of $\mathrm{Kd}$ then presented with complaints of fatigue, lethargy, cough and diarrhea. Laboratory tests were remarkable for acute renal failure, thrombocytopenia, and electrolyte abnormalities. Creatinine was severely elevated at $11 \mathrm{mg} / \mathrm{dL}$ (baseline of $0.67 \mathrm{mg} / \mathrm{dL}$ ), uric acid $15 \mathrm{mg} / \mathrm{dL}$, calcium 6.7 $\mathrm{mg} / \mathrm{dL}$, and phosphorus $5.6 \mathrm{mg} / \mathrm{dL}$. Hemoglobin was at her baseline of $11.5 \mathrm{gm} / \mathrm{dL}$, reticulocyte $1.78 \%$, normal bilirubin, and peripheral blood smear was unremarkable without schistocytes. Coagulation parameter prothrombin and activated thromboplastin time were normal.

She was suspected to have Tumor Lysis Syndrome (TLS) and assigned a Cairo-Bishop grade 4, as creatinine rose more than 6 times the baseline, indicating severe TLS. The patient was hydrated vigorously and given a dose of rasburicase. Table 1 represents biochemical markers of TLS at baseline and for the first four days of her hospital stay.

Table 1. Biochemical markers of TLS during admission

\begin{tabular}{|c|c|c|c|c|c|}
\hline Day & $\begin{array}{c}\text { Creatinine } \\
\mathrm{mg} / \mathrm{dL}\end{array}$ & $\begin{array}{c}\text { Phosphorus } \\
\mathrm{mg} / \mathrm{dL}\end{array}$ & $\begin{array}{c}\text { Potassium } \\
\mathrm{mEq} / \mathrm{L}\end{array}$ & $\begin{array}{c}\text { Calcium } \\
\mathrm{mg} / \mathrm{dL}\end{array}$ & $\begin{array}{c}\text { Uric acid } \\
\mathrm{mg} / \mathrm{dL}\end{array}$ \\
\hline Baseline & 0.67 & 3.3 & - & - & - \\
\hline Day 1 & 11.2 & 5.6 & 5.3 & 6.7 & 15 \\
\hline Day 2 & 13.4 & 5.3 & 5.4 & 6.4 & 14 \\
\hline Day 3 & 8.17 & 4.6 & 3.9 & 6.3 & 0.02 \\
\hline Day 4 & 7.47 & 3.2 & 4.3 & 7.1 & 0.02 \\
\hline
\end{tabular}

On hospital day 2, hemoglobin trended down to $9.9 \mathrm{~g} / \mathrm{dL}$ and haptoglobin was $46 \mathrm{mg} / \mathrm{dL}$. She was eventually started on dialysis on hospital day 3 due to worsening renal failure. The hemoglobin continued to fall steadily to $7.5 \mathrm{~g} / \mathrm{dL}, \mathrm{LDH}$ peaked at $3644 \mathrm{U} / \mathrm{L}$, haptoglobin decreased to $7 \mathrm{mg} / \mathrm{dL}$, White blood cells (WBC) $7^{\prime} 700$ cell $/ \mathrm{mL}$, platelets $69^{\prime} 000 \times 10^{9} / \mathrm{L}$ and peripheral blood smear revealed occasional schistocytes (3-4/HPF).

Table 2. Hemolysis parameters during admission

\begin{tabular}{|c|c|c|c|c|}
\hline Day & Hemoglobin g/dL & $\begin{array}{c}\text { Platelets x } \\
10^{9} / \mathrm{L}\end{array}$ & LDH U/L & $\begin{array}{c}\text { Haptoglobin } \\
\mathrm{mg} / \mathrm{dL}\end{array}$ \\
\hline Baseline & 11.6 & 141 & 250 & - \\
\hline Day 1 & 11.5 & 42 & 2460 & - \\
\hline Day 2 & 9.9 & 46 & 2500 & 46 \\
\hline Day 3 & 7.5 & 69 & 3644 & 24 \\
\hline Day 4 & 6.8 & 75 & 2300 & 7 \\
\hline
\end{tabular}

The development of anemia and thrombocytopenia with laboratory evidence of microangiopathic hemolysis raised the suspicion for carfilzomib induced-TMA. ADAMTS13 activity level was $48 \%$ which ruled out thrombotic thrombocytopenic purpura (TTP). Other causes of TMA such as disseminated intravascular coagulation, mechanical valve, and uncontrolled hypertension were ruled out. See Table 2 shows the initial and follow-up hemolysis parameters.

Hemodialysis and supportive care were continued though the patient's hospital course was complicated by oropharyngeal and gastrointestinal bleeding which was managed with a proton pump inhibitor continuous drip. One week after hospitalization, the platelets and hemoglobin improved to near baseline and the patient was eventually discharged. She unfortunately remained dependent on hemodialysis.

\section{Discussion}

TMA encompasses a spectrum of three different conditions: hemolytic uremic syndrome, atypical hemolytic uremic syndrome (aHUS) and TTP; sharing the triad of MAHA, thrombocytopenia and renal failure, but with different causation and presentation. Known etiologies for TMA can be hereditary and acquired; hereditary etiologies include ADAMTS13 deficiency and mutations affecting the alternative complement pathway. Acquired causes of TMA include infections, drug induced, and MM, which has been implicated as a cause of TMA particularly in cases of uncontrolled $\mathrm{MM}$ at the time of initial diagnosis. HSCT has also been associated with MAHA and TMA, classified under a category called transplant-associated thrombotic microangiopathy (TA-TMA) [10,11,12,13].

Drug induced TMA (DITMA) has been reported with the use of different medications such as quinines, clopidogrel, calcineurin inhibitors, cyclosporin, vascular endothelial growth factor (VEGF) inhibitors, and chemotherapeutic agents such as gemcitabine, mitomycin and proteasome inhibitors like the subject in our case, PI $[14,15,16]$. There are several postulated mechanisms of PI induced TMA. One hypothesis is based on immune-mediated toxicity, as PIs could lead to high levels of proinflammatory cytokines (including IL-6 and TNF-a), and create a microvascular milieu allowing for the formation of drug-dependent antibodies [17].

Another mechanism is dose-mediated toxicity involving VEGF inhibition leading to microvascular injury, particularly to glomerular capillaries. Similar to the mechanism by which bevacizumab (anti-VEGF antibodies), pazopanib (VEGF 1-3 inhibitor) and sunitinib (VEGF tyrosine kinase inhibitor) can lead to TMA-related kidney damage specifically via podocyte injury, causing proteinuria and hypertension $[14,18,19]$.

Our literature review using PubMed with the keywords: carfilzomib, bortezomib, proteasome inhibitors, TMA, thrombotic, microangiopathy, HUS, AHUS and TTP revealed reports 19 cases of DITMA attributed to PI use between 2008-2018 (Table 3). In 2016, Yui et al [20] reviewed an additional 11 cases at six major institutions in US and Europe which were not included because the individual presentation and outcome for each case was not specified. Ixazomib, the most recent PI has also been linked to DITMA, and 3 reported cases are included in Table 3 . 
Table 3. Reported cases of TMA attributed to PI use from 2008 to 2018. LGIB: Lower Gastrointestinal bleeding. HD: Hemodialysis. PLEX: Plasma exchange. Trfx: Transfusion. FFP: Fresh Frozen Plasma. HTN: Hypertension

\begin{tabular}{|c|c|l|c|l|l|c|c|}
\hline No. & Age \& sex & PI used & Time of onset & Presenting symptoms & Treatment & Outcome & Ref \\
\hline 1 & $61 \mathrm{~F}$ & Bortezomib & C5 D20 & Profuse diarrhea and LGIB & HD & Recovered & {$[21]$} \\
\hline 2 & $70 \mathrm{~F}$ & Bortezomib & C3 D2 & Weakness and pigmenturia & PLEX \& Trfx & Recovered & {$[22]$} \\
\hline 3 & $52 \mathrm{~F}$ & Bortezomib & C5 D11 & Diarrhea and weakness & PLEX \& Trfx & Partial recovery & {$[23]$} \\
\hline 4 & $57 \mathrm{~F}$ & Bortezomib & C1 D2 & Weakness and Hemoglobinurea & Trfx & Recovered & {$[17]$} \\
\hline 5 & $54 \mathrm{M}$ & Bortezomib & C1 D8 & Weakness and petechiae & FFP & Recovered & {$[24]$} \\
\hline 6 & $62 \mathrm{M}$ & Carfilzomib & C2 D14 & Edema, HTN \& proteinurea & Discontinue & Death & {$[9]$} \\
\hline 7 & $63 \mathrm{M}$ & Carfilzomib & C1 D14 & Oligurea, Edema \& weakness & PLEX \& Trfx & Partial recovery & {$[25]$} \\
\hline 8 & $74 \mathrm{M}$ & Carfilzomib & C1 D15 & Edema, hematoma \& lethargy & PLEX & Partial recovery & {$[26]$} \\
\hline 9 & $73 \mathrm{M}$ & Carfilzomib & C2 & Weakness and HTN & PLEX & Partial recovery & {$[27]$} \\
\hline 10 & $72 \mathrm{~F}$ & Carfilzomib & C6 & Diarrhea, vomiting \& weakness & PLEX \& Trfx & Recovered & {$[27]$} \\
\hline 11 & $61 \mathrm{~F}$ & Carfilzomib & C9 D5 & Fatigue \& dyspnea & PLEX, HD, Trfx \& eculizumab & Recovered & {$[28]$} \\
\hline 12 & $70 \mathrm{M}$ & Carfilzomib & C2 D6 & Diarrhea & Discontinue & Recovered & {$[29]$} \\
\hline 13 & $66 \mathrm{~F}$ & Carfilzomib & C2 D8 & Weakness and cough & HD & Recovered & {$[29]$} \\
\hline 14 & $63 \mathrm{M}$ & Carfilzomib & C2 D15 & Fever, diarrhea \& cough & Discontinue & Recovered & {$[29]$} \\
\hline 15 & $58 \mathrm{M}$ & Carfilzomib & C3 D7 & Fever \& oliguria & HD & Recovered & {$[29]$} \\
\hline 16 & $62 \mathrm{~F}$ & Carfilzomib & C1 D1 & Fever \& weakness & PLEX and steroids & Death & {$[30]$} \\
\hline 17 & $51 \mathrm{M}$ & Bortezomib & C1 D21 & Proteinurea, skin lesions & PLEX \& HD & Partial recovery & {$[31]$} \\
\hline 18 & $75 \mathrm{~F}$ & Ixazomib & C1 D15 & Vomiting, diarrhea \& weakness & PLEX \& Rituximab & Recovered & {$[32]$} \\
\hline 19 & $71 \mathrm{~F}$ & Ixazomib & C2 D28 & Fever, diarrhea \& weakness & PLEX \& FFP & Recovered & {$[33]$} \\
\hline
\end{tabular}

Similar to our patient, greater than $90 \%$ of the patients reviewed had acute kidney injury and more than half of the cases reported complaints of fatigue and lethargy likely due to the second most common presenting symptoms of diarrhea and vomiting. Onset of DITMA after PI exposure varied widely in the described cases ranging from hours up to 17 months, with a median of 46 days. Our case is somewhat unique due to the very late onset of presentation of TMA after receiving carfilzomib for 23 months. To the best of our knowledge, presentation this far out from initiation of treatment has not been reported.

Most of the reported cases of PI induced TMA were managed with plasma exchange, transfusion, and dialysis. Twelve of 19 cases achieved full recovery with various therapeutic measures; hemodialysis, plasmapheresis, discontinuing the PI, and supportive transfusion. Two patients achieved full recovery with combined measures: one received plasmapheresis, hemodialysis and eculizumab, and the other achieved full recovery with plasmapheresis and rituximab. With the unpredictable responses to various therapeutic interventions, we are unable to draw any conclusions regarding treatment efficacy and further understanding of the pathophysiology of DITMA should be investigated.

Resolution of the DITMA after hemodialysis might support the dose-dependent toxicity of the PI driving the DITMA, while its resolution after plasmapheresis, eculizumab and rituximab support the theory of an immune mediated mechanism driving DITMA.

Eculizumab is a monoclonal antibody against complement protein $\mathrm{C5}$ that inhibits the formation of $\mathrm{C} 5 \mathrm{a}$, a proinflammatory cytokine. Complement targeted therapy with eculizumab is a well-established treatment for aHUS. Two prospective studies published in 2013 concluded that eculizumab is associated with significant time-dependent improvement in renal function in patients with atypical HUS [34]. In our review, eculizumab was given resulting in positive outcomes in two reported cases of DITMA [28] which may have contributed to a shortened hospital-stay and faster recovery of cytopenias within 3-5 weeks. However, its use remains off-label and there remains no standard of care for PI-induced TMA. The mechanism of eculizumab might provide us with better understanding of the immunological response in drug-induced TMA, but this remains an area for further study.

Carfilzomib is rapidly becoming a corner-stone treatment in MM. Despite its widespread use, the precise incidence of carfilzomib-induced TMA remains unknown. Although TMA was not reported with the initial trials [35], it is now a recognized adverse effect of carfilzomib. Reporting adverse effects in case series helps expand our knowledge of the risks and benefits of treatments. Quick recognition of these symptoms and laboratory results in respect to their temporal relationship to the initiation of PI can also save patients from potential fatal side effects, which could be reversible.

Finally, our case highlights the need for vigilant monitoring and the importance of clinical acumen and history taking in patients at risk for TMA, even after extended exposures to known causative drugs.

\section{Acknowledgements}

This work is supported, in part, by the efforts of Dr. Moro O. Salifu M.D., M.P.H., M.B.A., M.A.C.P., Professor and Chairman of Medicine through NIH Grant number S21MD012474.

\section{References}

[1] Siegel, R.L., K.D. Miller, and A. Jemal, Cancer statistics, 2015. CA Cancer J Clin, 2015. 65(1): p. 5-29.

[2] Ludwig, H., et al., International Myeloma Working Group recommendations for global myeloma care. Leukemia, 2014. 28(5): p. 981-92. 
[3] Palumbo, A. and K. Anderson, Multiple myeloma. N Engl J Med, 2011. 364(11): p. 1046-60.

[4] Adams, J. and M. Kauffman, Development of the proteasome inhibitor Velcade (Bortezomib). Cancer Invest, 2004. 22(2): p. 304-11.

[5] Siegel, D.S., et al., A phase 2 study of single-agent carfilzomib (PX-171-003-A1) in patients with relapsed and refractory multiple myeloma. Blood, 2012. 120(14): p. 2817-25.

[6] Dimopoulos, M.A., et al., Carfilzomib and dexamethasone versus bortezomib and dexamethasone for patients with relapsed or refractory multiple myeloma (ENDEAVOR): a randomised, phase 3, open-label, multicentre study. Lancet Oncol, 2016. 17(1): p. 27-38.

[7] Chari, A. and D. Hajje, Case series discussion of cardiac and vascular events following carfilzomib treatment: possible mechanism, screening, and monitoring. BMC Cancer, 2014. 14: p. 915.

[8] Harvey, R.D., Incidence and management of adverse events in patients with relapsed and/or refractory multiple myeloma receiving single-agent carfilzomib. Clin Pharmacol, 2014. 6: p. 87-96.

[9] Hobeika, L., S.E. Self, and J.C. Velez, Renal thrombotic microangiopathy and podocytopathy associated with the use of carfilzomib in a patient with multiple myeloma. BMC Nephrol, 2014. 15: p. 156.

[10] Noris, M. and G. Remuzzi, Atypical hemolytic-uremic syndrome. N Engl J Med, 2009. 361(17): p. 1676-87.

[11] Noris, M., et al., Relative role of genetic complement abnormalities in sporadic and familial aHUS and their impact on clinical phenotype. Clin J Am Soc Nephrol, 2010. 5(10): p. 1844-59.

[12] Roy, V., et al., Thrombotic thrombocytopenic purpura-like syndromes following bone marrow transplantation: an analysis of associated conditions and clinical outcomes. Bone Marrow Transplant, 2001. 27(6): p. 641-6.

[13] Besbas, N., et al., A classification of hemolytic uremic syndrome and thrombotic thrombocytopenic purpura and related disorders. Kidney Int, 2006. 70(3): p. 423-31.

[14] Eremina, V., et al., VEGF inhibition and renal thrombotic microangiopathy. N Engl J Med, 2008. 358(11): p. 1129-36.

[15] Reese, J.A., et al., Drug-induced thrombotic microangiopathy: Experience of the Oklahoma Registry and the BloodCenter of Wisconsin. Am J Hematol, 2015. 90(5): p. 406-10.

[16] Al-Nouri, Z.L., et al., Drug-induced thrombotic microangiopathy: a systematic review of published reports. Blood, 2015. 125(4): p. 616-8.

[17] Moore, H. and K. Romeril, Multiple myeloma presenting with a fever of unknown origin and development of thrombotic thrombocytopenic purpura post-bortezomib. Intern Med J, 2011. 41(4): p. 348-50.

[18] Eremina, V. and S.E. Quaggin, Biology of anti-angiogenic therapy-induced thrombotic microangiopathy. Semin Nephrol, 2010. 30(6): p. 582-90.
[19] Usui, J., et al., Clinicopathological spectrum of kidney diseases in cancer patients treated with vascular endothelial growth factor inhibitors: a report of 5 cases and review of literature. Hum Pathol, 2014. 45(9): p. 1918-27.

[20] Yui, J.C., et al., Proteasome inhibitor associated thrombotic microangiopathy. Am J Hematol, 2016. 91(9): p. E348-52.

[21] Chan, K.L., et al., Thrombotic microangiopathy complicating bortezomib-based therapy for multiple myeloma. Leuk Lymphoma, 2015. 56(7): p. 2185-6.

[22] Mehta, N., A. Saxena, and R. Niesvizky, Bortezomib-induced thrombotic thrombocytopaenic purpura. BMJ Case Rep, 2012. 2012.

[23] Salmenniemi, U. and K. Remes, Thrombotic microangiopathy associated with bortezomib treatment in a patient with relapsed multiple myeloma. Hematol Rep, 2012. 4(2): p. e13.

[24] Morita, R., et al., Thrombotic microangiopathy after treatment with bortezomib and dexamethasone in a patient with multiple myeloma. Int J Hematol, 2008. 88(2): p. 248-250.

[25] Lodhi, A., et al., Thrombotic microangiopathy associated with proteasome inhibitors. Clin Kidney J, 2015. 8(5): p. 632-6.

[26] Sullivan, M.R., et al., Carfilzomib associated thrombotic microangiopathy initially treated with therapeutic plasma exchange. J Clin Apher, 2015. 30(5): p. 308-10.

[27] Qaqish, I., et al., Carfilzomib: A cause of drug associated thrombotic microangiopathy. Transfus Apher Sci, 2016. 54(3): p. 401-4.

[28] Gosain, R., et al., Gemcitabine and carfilzomib induced thrombotic microangiopathy: eculizumab as a life-saving treatment. Clin Case Rep, 2017. 5(12): p. 1926-1930.

[29] Chen, Y., et al., Thrombotic microangiopathy during carfilzomib use: case series in Singapore. Blood Cancer Journal, 2016. 6: p. e450.

[30] Atrash, S., et al., Fatal Thrombotic Microangiopathy Developing within 24 Hours of Carfilzomib in a Patient with Relapsed Multiple Myeloma (MM). Blood, 2012. 120(21): p. 5037-5037.

[31] Van Keer, J., et al., Renal Thrombotic Microangiopathy Associated with the Use of Bortezomib in a Patient with Multiple Myeloma. Case Rep Hematol, 2016. 2016: p. 6020691.

[32] Yui, J.C., A. Dispenzieri, and N. Leung, Ixazomib-induced thrombotic microangiopathy. Am J Hematol, 2017. 92(4): p. E53e55.

[33] Atallah-Yunes, S.A. and M.H. Soe, Drug-Induced Thrombotic Microangiopathy due to Cumulative Toxicity of Ixazomib. Case Rep Hematol, 2018. 2018: p. 7063145.

[34] Legendre, C.M., et al., Terminal complement inhibitor eculizumab in atypical hemolytic-uremic syndrome. N Engl J Med, 2013. 368(23): p. 2169-81.

[35] Stewart, A.K., et al., Carfilzomib, lenalidomide, and dexamethasone for relapsed multiple myeloma. $\mathrm{N}$ Engl J Med, 2015. 372(2): p. 142-52.

C The Author(s) 2019. This article is an open access article distributed under the terms and conditions of the Creative Commons Attribution (CC BY) license (http://creativecommons.org/licenses/by/4.0/). 